PROCEEDINGS OF THE

AMERICAN MATHEMATICAL SOCIETY

Volume 139, Number 5, May 2011, Pages 1751-1761

S 0002-9939(2010)10634-2

Article electronically published on October 20, 2010

\title{
BOUNDED TURNING CIRCLES ARE WEAK-QUASICIRCLES
}

\author{
DANIEL MEYER
}

(Communicated by Mario Bonk)

\begin{abstract}
We show that a metric Jordan curve $\Gamma$ is bounded turning if and only if there exists a weak-quasisymmetric homeomorphism $\varphi: \mathrm{S}^{1} \rightarrow \Gamma$.
\end{abstract}

\section{INTRODUCTION}

A metric Jordan curve $\Gamma$ is bounded turning (or $C$-bounded turning) if there is a constant $C \geq 1$ such that for each pair of points $x, y \in \Gamma$, the arc of smaller diameter $\Gamma[x, y] \subset \Gamma$ between $x, y$ satisfies

$$
\operatorname{diam} \Gamma[x, y] \leq C|x-y| .
$$

Here and in the following, we denote metrics by the Polish notation, i.e., by $|x-y|$. A homeomorphism of metric spaces $\varphi: X \rightarrow Y$ is called a weak-quasisymmetry (or $H$-weak-quasisymmetry) if there is a constant $H \geq 1$ such that

$$
|x-y| \leq|x-z| \Rightarrow|f(x)-f(y)| \leq H|f(x)-f(z)|,
$$

for all $x, y, z \in X$. In the present paper, we prove the following theorem.

Theorem 1.1. A metric Jordan curve $\Gamma$ is bounded turning if and only if there exists a weak-quasisymmetric homeomorphism $\varphi: \mathrm{S}^{1} \rightarrow \Gamma$.

The same proof shows the following.

Corollary 1.2. A metric Jordan arc $A$ is bounded turning if and only if there is a weak-quasisymmetric homeomorphism $\varphi:[0,1] \rightarrow A$.

1.1. Background. The following notion is closely related to weak-quasisymmetry. A homeomorphism $\varphi: X \rightarrow Y$ of metric spaces is called a quasisymmetry if there exists a homeomorphism $\eta:[0, \infty) \rightarrow[0, \infty)$ such that

$$
|x-y| \leq t|x-z| \Rightarrow|\varphi(x)-\varphi(y)| \leq \eta(t)|\varphi(x)-\varphi(z)|
$$

for all points $x, y, z \in X$ and $t \in[0, \infty)$. General background on (weak-)quasisymmetries can be found in Hei01.

Every quasisymmetry is a weak-quasisymmetry (pick $H=\eta(1)$ ). While the reverse does not hold in general, it is true in many practically relevant situations.

Received by the editors March 30, 2010 and, in revised form, May 22, 2010.

2010 Mathematics Subject Classification. Primary 30C65; Secondary 51F99.

Key words and phrases. Quasisymmetry, weak-quasisymmetry, bounded turning, weakquasicircle.

The author's research was supported by the Academy of Finland, projects SA-134757 and SA-118634.

(C) 2010 American Mathematical Society

Reverts to public domain 28 years from publication 
Recall that a metric space is doubling if there is a constant $N$ such that every ball of radius $r$ can be covered by at most $N$ balls of radius $r / 2$. Note that every Jordan curve $\Gamma \subset \mathbb{R}^{n}$ is doubling.

Theorem 1.3 ([Hei01, Theorem 10.19]). If $X$ is connected and both $X, Y$ are doubling, then every weak-quasisymmetry $\varphi: X \rightarrow Y$ is quasisymmetric.

Definition (1.3) for quasisymmetry appears in TV80. In earlier work (for example in AB56, Ahl63]) quasisymmetry is defined by (1.2); it is however only applied to maps where the two notions agree by the theorem cited above.

A quasicircle is the image of the unit circle $S^{1}$ by a quasisymmetric map. Ahlfors has given in Ahl63 the following geometric characterization for planar quasicircles. For a Jordan curve $\Gamma \subset \mathbb{C}$ it holds that

$\Gamma$ is a quasicircle $\Leftrightarrow \Gamma$ is bounded turning.

Tukia and Väisälä generalize this characterization to all metric Jordan curves in TV80, namely for a metric Jordan curve $\Gamma$ it holds that

$\Gamma$ is a quasicircle $\Leftrightarrow \Gamma$ is bounded turning and doubling.

If we call the weak-quasisymmetric image of the unit circle $S^{1}$ a weak-quasicircle, then Theorem 1.1 may be expressed as follows. For a Jordan curve $\Gamma$ it holds that

$\Gamma$ is a weak-quasicircle $\Leftrightarrow \Gamma$ is bounded turning.

It is easy to see that the quasisymmetric image of a doubling space is doubling (see Hei01, Theorem 10.18]). Thus one recovers from Theorem 1.1 together with Theorem 1.3 the Tukia-Väisälä characterization of quasicircles.

The first example of a bounded turning circle that is not a quasicircle was given by Tukia-Väisälä in TV80, Example 4.12]. A simple catalog $\mathcal{S}$ of bounded turning circles that includes a bi-Lipschitz copy of any bounded turning circle is given in $[\mathrm{HM}$. A curve $S \in \mathcal{S}$ from this catalog is doubling, i.e., a quasicircle, if and only if a simple condition is satisfied.

1.2. Organization of the paper. The "if"-part of Theorem 1.1 is trivial. Namely, let $\varphi: \mathrm{S}^{1} \rightarrow \Gamma$ be $H$-weak-quasisymmetric. Consider arbitrary points $a, b \in \mathrm{S}^{1}$, and let $[a, b] \subset \mathrm{S}^{1}=[0,1] /\{0 \sim 1\}$ be the arc between $a$ and $b$ of smaller diameter. Then for points $x, y \in[a, b]$ it holds that

$$
|\varphi(x)-\varphi(y)| \leq|\varphi(x)-\varphi(a)|+|\varphi(a)-\varphi(y)| \leq 2 H|\varphi(a)-\varphi(b)|
$$

or, assuming $a \leq x \leq y \leq b$,

$$
|\varphi(x)-\varphi(y)| \leq H|\varphi(x)-\varphi(b)| \leq H^{2}|\varphi(a)-\varphi(b)| .
$$

Therefore $\operatorname{diam} \varphi([a, b]) \leq C|\varphi(a)-\varphi(b)|$, where $C=\min \left\{2 H, H^{2}\right\}$. Thus $\Gamma$ is $C$-bounded turning.

The rest of this paper concerns the construction of a weak-quasisymmetry $\varphi: S^{1}$ $\rightarrow \Gamma$ for a given bounded turning circle $\Gamma$. In Section 2 we show that we can restrict our attention to the case when $\Gamma$ is 1-bounded turning. Also, an elementary lemma about dividing arcs into subarcs of equal diameter is proved.

In Section 3 we divide $\Gamma$ into $\operatorname{arcs} \Gamma_{1}^{n}, \ldots, \Gamma_{N^{n}}^{n}($ for each $n \in \mathbb{N})$. Two $\operatorname{arcs} \Gamma_{i}^{n}, \Gamma_{j}^{n}$ have roughly the same diameter. Each $\operatorname{arc} \Gamma_{i}^{n+1}$ is contained in a (unique) $\operatorname{arc} \Gamma_{j}^{n}$; thus the sets $\Gamma^{n}=\left\{\Gamma_{j}^{n} \mid j=1, \ldots, N^{n}\right\}$ form subdivisions of $\Gamma$. 
In Section 4 we divide the unit circle $\mathrm{S}^{1}$ into intervals $I_{1}^{n}, \ldots, I_{N^{n}}^{n}$. Neighboring intervals $I_{j}^{n}, I_{j+1}^{n}$ have roughly the same diameter. Furthermore, the combinatorics of the subdivisions of $\Gamma$ and $\mathrm{S}^{1}$ is the same, namely $\Gamma_{i}^{n+1} \subset \Gamma_{j}^{n} \Leftrightarrow I_{i}^{n+1} \subset I_{j}^{n}$.

The map $\varphi: S^{1} \rightarrow \Gamma$ is defined in Section 5 by mapping endpoints of intervals $I_{j}^{n}$ to endpoints of corresponding $\operatorname{arcs} \Gamma_{j}^{n}$.

Section 6 and Section 7 are preparations to prove the weak-quasisymmetry of $\varphi$. Namely, we show that the diameter of any interval in $S^{1}$ can be estimated in terms of the subdivision-intervals $I_{j}^{n}$. Then we show that if $I_{i}^{n}, I_{j}^{m}$ are the largest subdivision-intervals contained in adjacent intervals of the same length, then $|m-n|$ is bounded.

Section 8 finishes the proof of Theorem 1.1 .

1.3. Notation. The unit circle is denoted by $\mathrm{S}^{1}$, which we identify with $[0,1] /$ $\{0 \sim 1\}$. The unit circle is thus equipped with the orientation inherited from the real line. We always assume that $S^{1}$ is equipped with the arc-length metric denoted by $\lambda(s, t)$; i.e., if $0 \leq s \leq t \leq 1$, then

$$
\lambda(s, t)=\min \{|t-s|,|s+(1-t)|\} .
$$

The diameter with respect to this metric of an interval $I \subset S^{1}=[0,1] /\{0 \sim 1\}$ is denoted by $|I|$. Note that $|I|$ equals the Lebesgue measure of $I$ in the case when $|I| \leq\left|\mathrm{S}^{1} \backslash I\right|$.

\section{Preliminaries}

We first show that we can restrict our attention to 1-bounded turning circles. More precisely, we show that any bounded turning circle is bi-Lipschitz equivalent to a 1-bounded turning circle.

Then we prove that any arc can be divided into subarcs of equal diameter.

2.1. Diameter distance. Given any metric Jordan curve or Jordan $\operatorname{arc} \Gamma$ we define the diameter distance on $\Gamma$ by

$$
\operatorname{dd}(x, y):=\operatorname{diam} \Gamma[x, y]
$$

for all $x, y \in \Gamma$, where $\Gamma[x, y] \subset \Gamma$ is the arc of smaller diameter between $x, y$. We record some properties of $\mathrm{dd}$.

\section{Lemma 2.1.}

(1) $\mathrm{dd}$ is a metric on $\Gamma$.

(2) $\Gamma$ is $C$-bounded turning if and only if id: $\Gamma \rightarrow(\Gamma$, dd $)$ is $C$-bi-Lipschitz.

(3) For any arc $A \subset \Gamma$ it holds that

$$
\operatorname{diam}_{\mathrm{dd}} A=\operatorname{diam} A .
$$

Here diam $_{\mathrm{dd}}$ denotes the diameter with respect to $\mathrm{dd}$.

(4) $(\Gamma, \mathrm{dd})$ is 1-bounded turning.

Proof. (1) is elementary.

To prove (3), first observe that for all $x, y \in A,|x-y| \leq \mathrm{dd}(x, y)$, so $\operatorname{diam} A \leq$ $\operatorname{diam}_{\mathrm{dd}} A$. Next, for all $x, y \in A, \operatorname{dd}(x, y) \leq \operatorname{diam} A$, so $\operatorname{diam}_{\mathrm{dd}} A \leq \operatorname{diam} A$.

In the following, $\Gamma[x, y] \subset \Gamma$ will always denote the arc of smaller diameter between points $x, y \in \Gamma$. Property (4) follows directly from (3), since $\mathrm{dd}(x, y)=$ $\operatorname{diam} \Gamma[x, y]=\operatorname{diam}_{\mathrm{dd}} \Gamma[x, y]$ for all $x, y \in \Gamma$. 
It remains to establish (2). If $\Gamma$ is $C$-bounded turning, then for all $x, y \in \Gamma$,

$$
\mathrm{dd}(x, y)=\operatorname{diam}(\Gamma[x, y]) \leq C|x-y| \leq C \mathrm{dd}(x, y) .
$$

Thus the identity map id: $\Gamma \rightarrow(\Gamma, \mathrm{dd})$ is $C$-bi-Lipschitz. Conversely, if this map is $C$-bi-Lipschitz, then for all $x, y \in \Gamma$,

$$
\operatorname{diam}(\Gamma[x, y])=\operatorname{diam}_{\mathrm{dd}}(\Gamma[x, y])=\mathrm{dd}(x, y) \leq C|x-y| .
$$

Therefore $(\Gamma,|\cdot|)$ is $C$-bounded turning.

It is elementary that postcomposing an $H$-weak-quasisymmetry with an $L$-biLipschitz map yields an $H L^{2}$-weak-quasisymmetry.

Assume we have constructed for a given bounded turning circle $\Gamma$ a weakquasisymmetry $\varphi: \mathrm{S}^{1} \rightarrow(\Gamma, \mathrm{dd})$. Then the composition $\mathrm{S}^{1} \stackrel{\varphi}{\rightarrow}(\Gamma, \mathrm{dd}) \stackrel{\text { id }}{\rightarrow} \Gamma$ is the desired weak-quasisymmetric parametrization of $\Gamma$. Thus to prove Theorem 1.1 it is enough to construct a weak-quasisymmetry $\varphi: S^{1} \rightarrow \Gamma$ for any 1-bounded turning circle $\Gamma$.

2.2. Dividing arcs. Here we prove that any metric Jordan arc can be divided into any given number of subarcs, each having exactly the same diameter.

The problem of finding points on a metric Jordan arc such that consecutive points are at the same distance is a non-trivial problem. In 1930 Menger gave a proof [Men30, p. 487]; that is short, simple, and natural, but wrong. It was proved for arcs in Euclidean space in AB35 and in the general case (indeed in more generality) in [Sch40, Theorem 3]; see also Väi82.

For the case at hand, i.e., for bounded turning arcs, it suffices to find subarcs that have equal diameter. We give the following elementary proof for this problem.

Lemma 2.2. Let $A$ be a metric Jordan arc and $N \geq 2$ an integer. Then we can divide $A$ into $N$ subarcs of equal diameter.

Proof. We may assume that $A$ is the unit interval $[0,1]$ equipped with some metric $d$. We claim that there are points $0=s_{0}<s_{1}<\cdots<s_{N-1}<s_{N}=1$ such that

$$
\operatorname{diam}\left[s_{0}, s_{1}\right]=\operatorname{diam}\left[s_{1}, s_{2}\right]=\cdots=\operatorname{diam}\left[s_{N-1}, s_{N}\right],
$$

where diam denotes diameter with respect to the metric $d$. When $N=2$ this follows by applying the intermediate value theorem to the function $[0,1] \ni s \mapsto$ $\operatorname{diam}[0, s]-\operatorname{diam}[s, 1]$.

According to Lemma 2.1 (3), we may measure the diameter with respect to the diameter distance. Thus, using Lemma 2.1 (44), we may assume that $A$ is 1-bounded turning, i.e., that for any $[s, t] \subset[0,1]$,

$$
d(s, t)=\operatorname{diam}[s, t] .
$$

Next we modify $d$ to get a metric $d_{\epsilon}$ that is strictly increasing in the sense that

$$
[s, t] \subsetneq\left[s^{\prime}, t^{\prime}\right] \subset[0,1] \Longrightarrow d_{\epsilon}(s, t)<d_{\epsilon}\left(s^{\prime}, t^{\prime}\right) .
$$

The crucial point here is the strict inequality, which need not hold in general. To this end, fix $\epsilon>0$, and for all $s, t \in[0,1]$ set

$$
d_{\epsilon}(s, t):=d(s, t)+\epsilon|t-s| .
$$

Then from (2.2) it follows that

$$
\operatorname{diam}_{\epsilon}[s, t]=\operatorname{diam}[s, t]+\epsilon|t-s|=d_{\epsilon}(s, t),
$$


where diam $_{\epsilon}$ denotes diameter with respect to $d_{\epsilon}$. This immediately implies (2.3).

We now show that $[0,1]$ can be divided into $N$ subintervals of equal $d_{\epsilon}$-diameter. Consider the compact set $S:=\left\{\mathbf{s}=\left(s_{1}, \ldots, s_{N-1}\right) \mid 0 \leq s_{1} \leq \cdots \leq s_{N-1} \leq 1\right\}$. Set $s_{0}:=0, s_{N}:=1$. The function $\varphi: S \rightarrow \mathbb{R}$ defined by

$$
\varphi(\mathbf{s}):=\max _{0 \leq i \leq N-1} \operatorname{diam}_{\epsilon}\left[s_{i}, s_{i+1}\right]-\min _{0 \leq j \leq N-1} \operatorname{diam}_{\epsilon}\left[s_{j}, s_{j+1}\right]
$$

assumes a minimum on $S$. If this minimum is zero, we are done. Otherwise, there are adjacent intervals $\left[s_{i-1}, s_{i}\right],\left[s_{i}, s_{i+1}\right]$ that have different $d_{\epsilon}$-diameter. Using the intermediate value theorem as before, we can find $s_{i}^{\prime} \in\left[s_{i-1}, s_{i+1}\right]$ such that $\operatorname{diam}_{\epsilon}\left[s_{i-1}, s_{i}^{\prime}\right]=\operatorname{diam}_{\epsilon}\left[s_{i}^{\prime}, s_{i+1}\right]$. Then from (2.3) it follows that

$$
\min _{0 \leq j<N} \operatorname{diam}_{\epsilon}\left[s_{j}, s_{j+1}\right]<\operatorname{diam}_{\epsilon}\left[s_{i-1}, s_{i}^{\prime}\right]=\operatorname{diam}_{\epsilon}\left[s_{i}^{\prime}, s_{i+1}\right]<\max _{0 \leq i<N} \operatorname{diam}_{\epsilon}\left[s_{i}, s_{i+1}\right] .
$$

Applying this procedure to all subintervals of maximal $d_{\epsilon}$-diameter we obtain a strictly smaller minimum for the function $\varphi$, which is impossible. Thus the minimum must be zero, and so we can subdivide $[0,1]$ into $N$ subintervals of equal $d_{\epsilon}$-diameter.

Now consider a sequence $\epsilon_{n} \searrow 0$, as $n \rightarrow \infty$. Let $s_{1}^{n}<\cdots<s_{N-1}^{n}$ be the points that divide [0,1] into $N$ subintervals of equal diameter with respect to $d_{\epsilon_{n}}$. We can assume that for all $1 \leq j<N$, all points $s_{j}^{n}$ converge to $s_{j}$ as $n \rightarrow \infty$. It follows that for all $1 \leq i, j<N$,

$$
\operatorname{diam}\left[s_{i}, s_{i+1}\right]=\lim _{n \rightarrow \infty} \operatorname{diam}_{\epsilon_{n}}\left[s_{i}^{n}, s_{i+1}^{n}\right]=\lim _{n \rightarrow \infty} \operatorname{diam}_{\epsilon_{n}}\left[s_{j}^{n}, s_{j+1}^{n}\right]=\operatorname{diam}\left[s_{j}, s_{j+1}\right],
$$

as desired.

The previous lemma is also true for metric Jordan curves $\Gamma$. In this case we are free to choose any point in $\Gamma$ to be an endpoint of one of the subarcs.

\section{Dividing $\Gamma$}

Consider a 1-bounded turning metric Jordan curve $\Gamma$. We fix a point $a_{0} \in \Gamma$ and an orientation of $\Gamma$.

For each $n \in \mathbb{N}$ we will divide $\Gamma$ into $\operatorname{arcs} \Gamma_{1}^{n}, \ldots, \Gamma_{N^{n}}^{n}$, labeled consecutively on $\Gamma$, such that $a_{0}$ is the common endpoint of $\Gamma_{1}^{n}, \Gamma_{N^{n}}^{n}$. The set of these arcs is denoted by $\boldsymbol{\Gamma}^{n}$. Here and in the following the upper index $n$ will denote the order of the subdivision. In particular $N^{1}, N^{2}, \ldots, N^{n}, \ldots$ will be some (increasing) sequence of positive integers, not a geometric sequence.

Lemma 3.1. There are divisions $\Gamma^{n}$ of $\Gamma$ as above with the following properties:

(1) $\boldsymbol{\Gamma}^{n+1}$ is a subdivision of $\boldsymbol{\Gamma}^{n}$. This means that every $\Gamma^{n+1} \in \Gamma^{n+1}$ is contained in a (unique) $\Gamma^{n} \in \Gamma^{n}$.

(2) The diameters of the arcs of the $n$-th subdivision are comparable; more precisely,

for all $\Gamma, \Gamma^{\prime} \in \Gamma^{n}$.

$$
\frac{1}{2} \leq \frac{\operatorname{diam} \Gamma}{\operatorname{diam} \Gamma^{\prime}} \leq 2
$$

(3) The diameters of the $n$-th and the $(n+1)$-th subdivision are comparable; more precisely,

$$
\frac{1}{16} \operatorname{diam} \Gamma^{n} \leq \operatorname{diam} \Gamma^{n+1} \leq \frac{1}{4} \operatorname{diam} \Gamma^{n}
$$

for all $\Gamma^{n+1} \in \Gamma^{n+1}$ and $\Gamma^{n} \in \Gamma^{n}$. 
The last property implies that each $\operatorname{arc} \Gamma^{n} \in \Gamma^{n}$ is subdivided into at least four $\operatorname{arcs} \Gamma^{n+1} \in \Gamma^{n+1}$.

Before we construct these divisions of $\Gamma$, i.e., prove the previous lemma, we need some preparation.

Lemma 3.2. Let $A$ be a 1-bounded turning arc, and let $0<\delta \leq \operatorname{diam} A$. For each $n$ we divide $A$ into $n$ arcs $A_{1}, \ldots, A_{n}$ of equal diameter (see Lemma 2.2). Let $n$ be the smallest integer such that $\operatorname{diam} A_{1}=\operatorname{diam} A_{2}=\cdots=\operatorname{diam} A_{n} \leq \delta$. Then $\operatorname{diam} A_{j} \geq \delta / 2$ for all $j=1, \ldots, n$.

Proof. Let $n$ be as in the statement. If $n=1$, then $\delta=\operatorname{diam} A$, and there is nothing to prove.

Assume now that $n \geq 2$. Assume that the statement is false. Then the subarcs of equal diameter $A_{1}, \ldots, A_{n}$ have common diameter diam $A_{j}<\delta / 2$.

Claim. Suppose $A$ is subdivided into $k$ subarcs $A_{1}^{\prime}, \ldots, A_{k}^{\prime}$ of equal diameter greater than $\delta$. Then $2 k+1 \leq n$.

Assuming the $A_{i}$ and the $A_{j}^{\prime}$ are ordered in the same order along $A$, we see that one needs at least $A_{1}, A_{2}, A_{3}$ to cover $A_{1}^{\prime}$. Similarly, at least the first five arcs $A_{1}, \ldots, A_{5}$ are needed to cover $A_{1}^{\prime} \cup A_{2}^{\prime}$. Inducting over the arcs $A_{1}^{\prime}, \ldots, A_{k}^{\prime}$ proves the claim.

We obtain a contradiction when we set $k=n-1$.

Proof of Lemma 3.1. We start by dividing $\Gamma$ into $\operatorname{arcs} \Gamma_{1}^{1}, \ldots, \Gamma_{N^{1}}^{1}$ of equal diameter, such that $\operatorname{diam} \Gamma / 8 \leq \operatorname{diam} \Gamma_{j}^{1} \leq \operatorname{diam} \Gamma / 4$ for all $j=1, \ldots, N^{1}$ using Lemma 2.2 and Lemma 3.2 , for some $N^{1} \in \mathbb{N}$. Here $a_{0}$ is the common endpoint of $\Gamma_{1}^{1}$ and $\Gamma_{N^{1}}^{1}$.

Assume $\Gamma$ has been divided into arcs $\Gamma_{1}^{n}, \ldots, \Gamma_{N^{n}}^{n}$ satisfying Lemma 3.1. In particular, $1 / 2 \leq \operatorname{diam} \Gamma_{i}^{n} / \operatorname{diam} \Gamma_{j}^{n} \leq 2$ for all $i, j \in\left\{1, \ldots, N^{n}\right\}$. Set $\delta=$ $\frac{1}{4} \min _{j} \operatorname{diam} \Gamma_{j}^{n}$. Using Lemma 2.2 and Lemma 3.2 we divide each arc $\Gamma^{n}=\Gamma_{i}^{n}$ into arcs $\Gamma_{1}^{n+1}, \ldots, \Gamma_{N}^{n+1}$ (here $\Gamma_{j}^{n+1}=\Gamma_{i, j}^{n+1}$ and $N=N_{i}^{n}$ ) of equal diameter such that

$$
\delta / 2 \leq \operatorname{diam} \Gamma_{1}^{n+1}=\cdots=\operatorname{diam} \Gamma_{N}^{n+1} \leq \delta .
$$

Let $\Gamma_{1}^{n+1}, \ldots, \Gamma_{N^{n+1}}^{n+1}$ be the set of all these arcs, labeled along $\Gamma$, such that $a_{0}$ is the common point of $\Gamma_{1}^{n+1}, \Gamma_{N^{n+1}}^{n+1}$. It is clear that these arcs satisfy the properties of Lemma 3.1.

Thus the $\operatorname{arcs} \Gamma_{1}^{n}, \ldots, \Gamma_{N^{n}}^{n}$ have been constructed for all $n$.

\section{Dividing the Unit CiRCle}

For each $n \in \mathbb{N}$ we divide the unit circle $S^{1}=[0,1] /\{0 \sim 1\}$ into intervals $I_{1}^{n}, \ldots, I_{N^{n}}^{n}$, labeled consecutively on $\mathrm{S}^{1}$. The common endpoint of $I_{1}^{n}$ and $I_{N^{n}}^{n}$ is 0 . The set of these intervals is denoted by $\mathbf{I}^{n}$.

Lemma 4.1. There are divisions $\mathbf{I}^{n}$ of the unit circle $\mathrm{S}^{1}$ as above satisfying the following:

(1) $\mathbf{I}^{n+1}$ is a subdivision of $\mathbf{I}^{n}$. This means that every $I^{n+1} \in \mathbf{I}^{n+1}$ is contained in a (unique) interval $I^{n} \in \mathbf{I}^{n}$.

Two adjacent intervals $I, I^{\prime} \in \mathbf{I}^{n}$ are called neighbors (i.e., $I=I_{j}^{n}, I^{\prime}=I_{j+1}^{n}$ ). Note that neighbors are always elements of the same subdivision $\mathbf{I}^{n}$. 


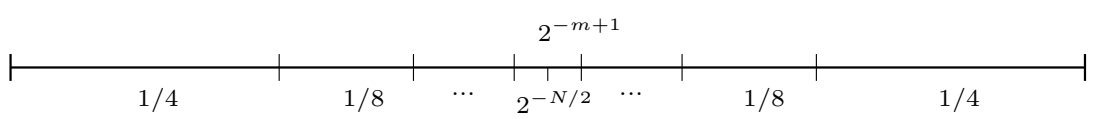

FiguRE 1. Subdividing an interval.

(2) The diameter of neighboring intervals are comparable. More precisely, they agree or differ by the factor 2 ,

$$
|I| /\left|I^{\prime}\right| \in\{1 / 2,1,2\},
$$

for all neighbors $I, I^{\prime}$.

(3) If $I_{i}^{n+1} \subset I_{j}^{n}$, then $\left|I_{i}^{n+1}\right| \leq\left|I_{j}^{n}\right| / 4$ for all $i=1, \ldots, N^{n+1}, j=1, \ldots, N^{n}$.

(4) The subdivisions $\mathbf{I}^{n}$ have the same combinatorics as the subdivisions $\boldsymbol{\Gamma}^{n}$. Namely,

$$
\begin{aligned}
& I_{i}^{n+1} \subset I_{j}^{n} \quad \Leftrightarrow \quad \Gamma_{i}^{n+1} \subset \Gamma_{j}^{n} \\
& \text { for all } i=1, \ldots, N^{n+1}, j=1, \ldots, N^{n} .
\end{aligned}
$$

Proof. Let $I=I_{i}^{n}$ be given. Assume that the corresponding $\operatorname{arc} \Gamma^{n}=\Gamma_{i}^{n}$ is divided into $N=N_{i}^{n}$ arcs $\Gamma_{j}^{n+1}$. Note that by construction $N_{i}^{n} \geq 4$.

Let $c$ be the midpoint of the interval $I$ (i.e., $c=\frac{1}{2}(a+b)$ if $\left.I=[a, b]\right)$. It divides $I$ into the left and right half of $I$.

To simplify the discussion we assume that $|I|=1$. For the general case, if we write in the following "length of a subinterval is $1 / 4$ ", it has to be replaced by "length of a subinterval is $1 / 4 \cdot|I|$ ", and so on.

Case 1. $N$ is even.

Starting from the left endpoint of $I$, we divide the left half of $I$ into intervals of length $1 / 4,1 / 8, \ldots, 2^{-N / 2}$ (times the length of $I$ ). There is one remaining interval of length $2^{-N / 2}$, which is the last interval of the left half of $I$. The right half of the interval is divided in a symmetric fashion, meaning that starting from the right endpoint we divide the right half into intervals of length $1 / 4,1 / 8, \ldots, 2^{-N / 2+1}, 2^{-N / 2}$, $2^{-N / 2}$. See the bottom of Figure 1.

Case 2. $N=2 m-1$ is odd.

We divide $I$ into $N+1=2 m$ subintervals as in Case 1. We then take the union of the two middle subintervals, i.e., the two subintervals containing the midpoint $c$. Thus $I$ is divided into $N$ subintervals of lengths

$$
1 / 4,1 / 8, \ldots, 2^{-m+1}, 2^{-m}, 2^{-m+1}, 2^{-m}, 2^{-m+1}, \ldots, 1 / 8,1 / 4 .
$$

See the top of Figure 1

This finishes the division of $I$, thus of all $I_{i}^{n}$, into intervals. Thus all $I_{j}^{n}$ have been constructed for all $n \in \mathbb{N}$. It is clear that they satisfy the properties of Lemma 4.1 .

In Case 1 there are two subintervals of $I$ containing the midpoint of $I$; in Case 2 there is a single subinterval of $I$. Such a subinterval is called a middle subinterval of $I$.

\section{The WEAK QUASISYMmetry}

Let $s_{0}^{n}, \ldots, s_{N^{n}-1}^{n}$ be the endpoints of the intervals $I_{j}^{n}$ ordered increasingly on $\mathrm{S}^{1}=[0,1] /\{0 \sim 1\}, s_{0}^{n}=0$ for all $n \in \mathbb{N}$. Let $a_{0}^{n}, \ldots, a_{N^{n}-1}^{n}$ be the endpoints of 
the $\operatorname{arcs} \Gamma_{j}^{n}$. Then we define $\varphi\left(s_{j}^{n}\right)=a_{j}^{n}$. From Lemma 3.1 (11) and Lemma 4.1 (4) it follows that $\varphi$ is well defined; i.e., if $s_{i}^{n}=s_{j}^{m}$, then $\varphi\left(s_{i}^{n}\right)=a_{i}^{n}=a_{j}^{m}=\varphi\left(s_{j}^{m}\right)$.

We show uniform continuity of $\varphi$ on the set $\mathbf{s}=\left\{s_{j}^{n} \mid n \in \mathbb{N}, j=0, \ldots, N^{n}-1\right\}$. Let $\delta_{n}:=\min _{j}\left|I_{j}^{n}\right|$. Then if $\lambda(s, t) \leq \delta_{n} / 2$ for two points $s, t \in \mathbf{s}$ (recall from (1.5) that $\lambda$ is the metric on $\mathrm{S}^{1}$ ), then $s, t$ are contained in adjacent intervals $I_{j}^{n}, I_{j+1}^{n}$. Thus $\varphi(s), \varphi(t)$ are contained in adjacent $\operatorname{arcs} \Gamma_{j}^{n}, \Gamma_{j+1}^{n}$. Thus

$$
|\varphi(s)-\varphi(t)| \leq \operatorname{diam} \Gamma_{j}^{n}+\operatorname{diam} \Gamma_{j+1}^{n} \leq 2 \cdot 4^{-n} \operatorname{diam} \Gamma
$$

by Lemma 3.1 (3), showing uniform continuity of $\varphi$ on $\mathbf{s}$. Since this set is dense in $\mathrm{S}^{1}, \varphi$ extends continuously to $\mathrm{S}^{1}$. The surjectivity is clear, since the set $\left\{a_{j}^{n} \mid n \in\right.$ $\left.\mathbb{N}, j=0, \ldots, N^{n}-1\right\}$ is dense in $\Gamma$. Injectivity follows from the fact that disjoint sets $I_{i}^{n}, I_{j}^{n}$ are mapped to disjoint $\operatorname{arcs} \Gamma_{i}^{n}, \Gamma_{j}^{n}$. Thus $\varphi: \mathrm{S}^{1} \rightarrow \Gamma$ is a homeomorphism.

\section{Estimating INTERVALS}

Given an interval $[x, y] \subset \mathrm{S}^{1}$ we define

$$
\delta([x, y]):=\max \left\{\left|I_{j}^{n}\right| \mid I_{j}^{n} \subset[x, y]\right\} .
$$

Here the maximum is taken over $n \in \mathbb{N}$ and all intervals $I_{j}^{n} \in \mathbf{I}^{n}$ as defined in Section 4.

Lemma 6.1. Let $[x, y] \subset \mathrm{S}^{1}$ be any interval. Then

$$
\delta([x, y]) \leq|[x, y]| \leq 12 \delta([x, y]) .
$$

Furthermore, if the maximum in equation (6.1) is attained for an interval $I=I_{j}^{n} \in$ $\mathbf{I}^{n}$, then there are two intersecting (possibly identical) intervals $\hat{I}, \hat{J} \in \mathbf{I}^{n-1}$ such that

$$
I \subset[x, y] \subset \hat{I} \cup \hat{J} .
$$

Proof. Let $I=I_{j}^{n} \subset[x, y]$ be one interval where the maximum from (6.1) is attained, i.e., $|I|=\delta([x, y])$. The left inequality, i.e., $|I|=\delta([x, y]) \leq|[x, y]|$, is obvious. Let $\hat{I} \supset I$ be the parent of $I$, i.e., the unique interval $\hat{I} \in \mathbf{I}^{n-1}$ containing $I$. Assume that $\hat{I}$ was subdivided into $N$ intervals $I^{n} \in \mathbf{I}^{n}$. We consider several cases.

Case $1 .|I|=|\hat{I}| / 4$.

This can happen only in the following instances: either $I$ is the left- or rightmost interval in $\hat{I}$ (i.e., $I, \hat{I}$ share a boundary point) or $N$ is equal to 4 or 5 and $I$ contains the midpoint of $\hat{I}$.

If $[x, y] \subset \hat{I}$, we are done, since then $|[x, y]| \leq|\hat{I}|=4|I|=4 \delta([x, y])$. We set $\hat{J}:=\hat{I}$.

Assume that $[x, y] \not \subset \hat{I}$. This means that one endpoint of $\hat{I}$, without loss of generality the left endpoint, is an interior point of $[x, y]$. From the maximality of $I$ it follows that $y \in \hat{I}$. Consider the left neighbor $\hat{J} \in \mathbf{I}^{n-1}$ of $\hat{I}$. Note that $|\hat{J}| \geq \frac{1}{2}|\hat{I}|=2|I|$. Thus $\hat{J} \not \subset[x, y]$ by the maximality of $I$. Thus $[x, y] \subset \hat{J} \cup \hat{I}$. It holds that $|\hat{I}|=4|I|$ and $|\hat{J}| \leq 2|\hat{I}|=8|I|$ so that

$$
|[x, y]| \leq 12 \delta([x, y]) \text {. }
$$


Case 2. $N \geq 6$ is even and $I=I_{j}^{n}$ is a middle subinterval of $\hat{I}$ (i.e., contains the midpoint of $I$ ).

Then either both $I_{j-2}^{n}, I_{j+3}^{n}$ or both $I_{j-3}^{n}, I_{j+2}^{n}$ have diameter strictly larger than $I$. We can assume without loss of generality the former case. This means that $I$ is in the left half of $\hat{I}$ and that

$$
I_{j-2}^{n} \cup I_{j-1}^{n} \cup I_{j}^{n} \cup I_{j+1}^{n} \cup I_{j+2}^{n} \cup I_{j+3}^{n}
$$

cover $[x, y]$. Note that the total length of these sets is $8|I|$. Thus $\delta([x, y]) \leq|[x, y]| \leq$ $8 \delta([x, y])$.

Case 3. $N \geq 7$ is odd and $I_{j}^{n}$ is the middle subinterval of $\hat{I}$.

Similar to the preceding case, $I_{j-3}^{n}, I_{j+3}^{n}$ have twice the length of $I$; thus they are not contained in $[x, y]$ and

$$
[x, y] \subset I_{j-3}^{n} \cup \cdots \cup I_{j+3}^{n} .
$$

Note that the total length of these intervals is $8|I|$. This finishes the claim in this case.

Case 4. Remaining case.

One of the neighbors of $I=I_{j}^{n}$, without loss of generality the left neighbor $I_{j-1}^{n}$, has twice the length of $I$.

Furthermore, there is a subinterval $I_{j+k}^{n} \in \mathbf{I}^{n}$ of $\hat{I}$ that has the same length as $I$. It is symmetric to $I$ with respect to the midpoint of $\hat{I}$. Then $I_{j-1}^{n}, I_{j+k+1}^{n}$ have twice the length of $I$, thus are not contained in $[x, y]$. Thus

$$
[x, y] \subset I_{j-1}^{n} \cup I_{j}^{n} \cup \cdots \cup I_{j+k}^{n} \cup I_{j+k+1}^{n} .
$$

The total length of the right-hand side is $8|I|$, finishing the claim.

Note that in Case 2-Case 4 the subintervals that cover $[x, y]$ are all contained in the parent $\hat{I}$; we then set $\hat{J}:=\hat{I}$.

\section{Estimating ORDER}

Now consider two adjacent intervals (in $\mathrm{S}^{1}$ ) of the same length, i.e., $[x-t, x]$, $[x, x+t]$ for some $x \in \mathrm{S}^{1}$ and $0<t \leq 1 / 2$. Consider the largest subdivision intervals contained in $[x-t, x],[x, x+t]$, meaning we consider intervals $J^{m} \in \mathbf{I}^{m}, I^{n} \in \mathbf{I}^{n}$ such that

$$
\begin{array}{ll}
J^{m} \subset[x-t, x], & I^{n} \subset[x, x+t] \text { and } \\
\left|J^{m}\right|=\delta([x-t, x]), & \left|I^{n}\right|=\delta([x, x+t]) .
\end{array}
$$

We want to show that $n, m$ differ by at most a constant $k_{0}$ (in fact $k_{0}=4$ ). Before giving the detailed argument, let us quickly describe the idea. From Lemma 6.1] it follows that $\left|I^{n}\right|,\left|J^{m}\right|$ are comparable. Without loss of generality, we can assume that $n \leq m$. Let $J^{n} \in \mathbf{I}^{n}$ be the (unique) $n$-th order subdivision-interval containing $J^{m}$. If $m-n$ is large, then $\left|J^{n}\right|$ is large compared to $\left|J^{m}\right|$, and thus large compared to $\left|I^{n}\right|$. Then $J^{n}, I^{n}$ have to be far apart. This is impossible.

Lemma 7.1. In the setting as above it holds that $|m-n| \leq 4$.

Proof. As in the outline given above we assume that $n \leq m$, and let $J^{n} \in \mathbf{I}^{n}$ be the subdivision-interval containing $J^{m}$. If $m-n=k_{0}$, then $\left|J^{m}\right| \leq 4^{-k_{0}}\left|J^{n}\right|$ by Lemma 4.1 (3). 
Claim. Consider two intervals $I, I^{\prime} \in \mathbf{I}^{n}$ such that $\left|I^{\prime}\right| /|I| \geq 2^{i+1}$ for some $i \geq 1$. Then $\operatorname{dist}\left(I, I^{\prime}\right) \geq 2^{i}|I|$.

This is clear, since the interval between $I, I^{\prime}$ has to contain one of size $2^{i}|I|$ by Lemma 4.1 (2).

From Lemma 6.1 it follows that $|[x-t, x+t]| \leq 24\left|I^{n}\right|$. Thus it follows from the previous claim that $\left|J^{n}\right| /\left|I^{n}\right| \leq 2^{5}$. Indeed $\left|J^{n}\right| /\left|I^{n}\right| \geq 2^{6}$ implies by the claim that $\operatorname{dist}\left(J^{n}, I^{n}\right) \geq 2^{5}\left|I^{n}\right|=32\left|I^{n}\right|$, which is impossible. Thus by Lemma 6.1

$$
\frac{1}{12}\left|I^{n}\right| \leq \frac{1}{12}|[x, x+t]|=\frac{1}{12}|[x-t, x]| \leq\left|J^{m}\right| \leq 4^{-k_{0}}\left|J^{n}\right| \leq 4^{-k_{0}} 2^{5}\left|I^{n}\right| .
$$

We obtain a contradiction if we choose $k_{0}$ such that $4^{-k_{0}} 2^{5}<1 / 12$ or $k_{0} \geq 5$. This finishes the proof.

\section{Proof of the theorem}

After these preparations, we are ready to prove the main theorem.

Proof of Theorem 1.1. Recall from Section 2.1 that it is enough to prove the theorem in the case when $\Gamma$ is 1-bounded turning. This means that for any two points $x, y \in \Gamma$, the arc of smaller diameter $\Gamma[x, y] \subset \Gamma$ between $x, y$ satisfies $\operatorname{diam} \Gamma[x, y]=|x-y|$.

Thus it is enough to show that the $\operatorname{arcs} \varphi([x-t, x]), \varphi([x, x+t])$ have comparable diameter for all $x \in \mathrm{S}^{1}, 0<t \leq 1 / 2$. Let $I_{-} \in \mathbf{I}^{m}, I_{+} \in \mathbf{I}^{n}$ be the largest intervals contained in $[x-t, x],[x, x+t]$; i.e.,

$$
\begin{array}{ll}
I_{-} \subset[x-t, x], & I_{+} \subset[x, x+t] \text { and } \\
\left|I_{-}\right|=\delta([x-t, x]), & \left|I_{+}\right|=\delta([x, x+t]) .
\end{array}
$$

Let $\hat{I}_{-}, \hat{J}_{-} \in \mathbf{I}^{m-1}$ be the intervals that cover $[x-t, x]$ according to Lemma 6.1] Then

$$
\begin{aligned}
|\varphi(x-t)-\varphi(x)| & =\operatorname{diam} \varphi([x-t, x]) \leq \operatorname{diam} \varphi\left(\hat{I}_{-} \cup \hat{J}_{-}\right) \\
& \leq 32 \operatorname{diam} \varphi\left(I_{-}\right) \quad \text { by Lemma 3.1 (3) } \\
& \leq 32 \cdot 2 \cdot 16^{4} \operatorname{diam} \varphi\left(I_{+}\right)
\end{aligned}
$$

by Lemma 7.1. Lemma 3.1 (3), and Lemma 3.1 (2),

$$
\begin{aligned}
& \leq 32 \cdot 2 \cdot 16^{4} \operatorname{diam} \varphi([x, x+t]) \\
& =32 \cdot 2 \cdot 16^{4}|\varphi(x)-\varphi(x+t)| .
\end{aligned}
$$

This finishes the proof.

\section{Concluding Remarks}

It is natural to ask how small the involved constants can be chosen. In particular, how small can the constant $H \geq 1$ of the weak-quasisymmetric parametrization $\varphi: \mathrm{S}^{1} \rightarrow \Gamma$ for a given $C$-bounded turning circle be chosen? Recall from (1.4) that the image of the unit circle by an $H$-weak-quasisymmetry is $C$-bounded turning, where $C=\min \left\{2 H, H^{2}\right\}$. Thus it is natural to ask if any $C$-bounded turning circle admits an $H$-weak-quasisymmetric parametrization, where $H=\max \{C / 2, \sqrt{C}\}$. As a starting point one may ask if any 1-bounded turning circle admits a 1-weakquasisymmetric parametrization. 


\section{ACKNOWLEDGMENTS}

The question that is answered by Theorem 1.1 was posed by David Herron. Jussi Väisälä provided many helpful suggestions and references.

\section{REFERENCES}

[AB35] F. Alt and G. Beer, Der n-Gittersatz in Bogen, Ergebisse math. Koll. 6 (1935), 7.

[AB56] L. V. Ahlfors and A. Beurling, The boundary correspondence under quasiconformal mappings, Acta Math. 96 (1956), 125-142. MR0086869 (19:258c)

[Ahl63] L. V. Ahlfors, Quasiconformal reflections, Acta Math. 109 (1963), 291-301. MR0154978 $(27: 4921)$

[Hei01] J. Heinonen, Lectures on analysis on metric spaces, Universitext, Springer-Verlag, New York, 2001. MR.1800917(2002c:30028)

[HM] D. A. Herron and D. Meyer, Quasicircles and bounded turning circles modulo bi-Lipschitz maps, to appear in Rev. Mat. Iberoamericana.

[Men30] K. Menger, Untersuchungen über allgemeine Metrik, Math. Ann. 103 (1930), no. 1, 466-501. MR1512632

[Sch40] I. J. Schoenberg, On metric arcs of vanishing Menger curvature, Ann. of Math. (2) 41 (1940), 715-726. MR0002903 (2:130b)

[TV80] P. Tukia and J. Väisälä, Quasisymmetric embeddings of metric spaces, Ann. Acad. Sci. Fenn. Ser. A I Math. 5 (1980), no. 5, 97-114. MR.595180 (82g:30038)

[Väi82] J. Väisälä, Dividing an arc to subarcs with equal chords, Colloq. Math. 46 (1982), no. 2, 203-204. MR0678135 (84d:54056)

Department of Mathematics and Statistics, University of Helsinki, P.O. Box 68, FI-00014 University of Helsinki, Finland

E-mail address: dmeyermail@gmail.com 